\title{
The holes problem in wireless sensor netwoks by using the available energy fairly
}

\author{
ZohrehMousavinasab, Zahra Saadati \\ Department of Computer, Omidieh Branch, Islamic Azad University, Omidieh, Iran \\ zohreh.mousavinasab@iauo.ac.ir \\ zahra.saadati@iau.ac.ir
}

\begin{abstract}
We consider the holes problem in wireless sensor netwoks by using the available energy fairly. The holes problem occurs when the available energy of some sensors are exhausted while others have significant energy. In this circumstance, ehile the total available energy of the network is high, the network is no longer working. Such a problem mainly happen due to unbalanced energy consumpsion in clustering and communication protocols. In this paper, we deal with this problem and propose an data aggregation algorithm based on the well-known clustering algorithm LEACH and tree construction mechanisms. In proposed method we consider the available energy of each sensor node as a weigthing parameter in clustering phase and tree-construction phase. Experimental results show the ability of the proposed method to increase the life time of the network and to distribute the energy uniformly along the network.
\end{abstract}

\section{Indexing terms/Keywords}

Wireless Sensor Network, Clustering, Energy distribution, Minimum Spanning Tree.

\section{INTRODUCTION}

Wireless Sensor Networks area specific type of MANETnetworks composed of several small and economical sensor nodes capable of calculations and wireless communications with each other. These networks are generally used for aggregating data in areas where a user cannot be present. One of the main constraints with such networks is energy consumption which affects lifetime of WSNs. Through the typical WSN scenario, data are aggregated via sensor nodes from monitored area, and these sensed data are sent effectively to central base station. Often neighboring nodes cause correlation or data redundancy.So, it is better to combine the data which results in saving energy and bandwidth. Howerer in some applications other sensor nodes are able to directly communicate with central station, but too much energy is consumed to transmit data to long distances. Therefore, in most cases the nodes communicate with central station through neighboring or median nodes. In this case, each node must know which neighbors can better and from a more appropriate route to transmit the bundles to central station. The most important communication protocols used in reducing communication distance and data combining include clustering and tree based methods; clusters lead to distribution and balance of energy as well as reduction in transfer of data bundles to central station. However, developing cluster, optimal selection of cluster head and appropriate size of the cluster are challenges for this approach. In addition, in most existing clustering protocols, there is too much energy consumption during data transfer, due to the long distance between some cluster heads and central station [1-4]. This problem can be solved by collecting the data in WSNs by developing data clustering tree. This tree is often minimum spanning tree and root of this tree is considered to be the central station. In a tree structure, each node has a parent node for sending its data to it. Considering clustering and tree based methods in one unified method has been studied before. But one important issues regarding such a method is that its main concentration is on decreasing the energy consumption by considering the shortest route for data transmition and have no attention to the distribution of energy along the WSN (so no attention to the holes problem) [5-8]. Therefore the life time of the WSN can be decreased due to the holes problem however the total available energy of WSN is substantial. In this paper we study such a unified method while concerning the holes problem. The reminder of the paper is organized as follows. In Section 2 the system model and the proposed method is presented. In Section 3 the effectiveness of the proposed method is evaluated using simulation. Finally the paper is concluded in Section 4.

\section{PROPSED METHOD}

In this section we introduce our proposed metod. At first we review the well known LEACH algorithm which is the essential base of our proposed method. Then the proposed method is dicussed in detail. . This algorithm is similar to LEACH, but it uses two parameters to select head cluster; one parameter is less distance and the other is that the selected head cluster must have too much energy. Then, a data aggregation tree is created between head clusters, using minimum spanning tree considering the available energy of each cluster head inside the distance between the cluster heads and the base stations. In this hybrid method which is called Weighted-TLEACH, energy consumption during data transfer from head cluster to central station is reduced significantly. Also due to less number of head clusters, the problem of high depth is solved in aggregation tree.

\subsection{LEACH Protocol}

Low Energy Adaptive Clustering Hierarchy LEACH is one of old routing protocols proposed by Heinzelman in MIT university and one of the most popular routing hierarchy protocols for sensor networks. In this protocol selection of head cluster is random and nodes are classified according to power of received signal from the head cluster. In LEACH the nodes are organized in local clusters and one node is selected as head cluster. All non-head cluster nodes send their data to head cluster. When the head cluster receives all members of the cluster, signal processing function (like data 
aggregation) is done on them and sends them to central station. Therefore a head-cluster node requires much more energy than other cluster nodes [9].

LEACH operation is divided among the rounds. Each round is started with phase during which the cluster is formed. Then during stable mode phase, the data are sent from non-head cluster nodes to head cluster and then to central station. Time period of each two phases is one round. In the beginning of initiation phase through a distributed algorithm, it is decided that which node is head cluster and then the cluster is formed [9]. In stable phase, each cluster member node sends its data to head cluster in a particular time division. Head cluster node aggregates and compresses the data received from cluster members and finally sends them to central station. In order to save in costs, head cluster is changed periodically among cluster nodes.

\subsection{The radio model used for calculating energy in send/receive of data packages}

All the collected data by sensors should be transferred to a data aggregation center. Longer distances consume more energy for sending data. It is estimated that for sending a $\mathrm{K}$ bit message in a route of $\mathrm{d}$ length, the consumed energy can be given as below [10]:

$$
\begin{array}{r}
E_{T X}(l, d)=E_{T X}-\operatorname{elec}(l)+E_{T X}-\operatorname{amp}(l, d)(1) \\
= \begin{cases}l \times E_{\text {elec }}+l \times \varepsilon_{f s} \times d^{2}, & d<d_{0} \\
l \times E_{\text {elec }}+l \times \varepsilon_{m p} \times d^{4}, & d>d_{0}\end{cases}
\end{array}
$$

Where Eelec is the electromagnetic loss and Eamp is loss of energy for reinforcing the sending. Threshold value of distance $\left(d_{0}\right)$ is obtained using equation (2) [10]:

$$
d_{0}=\sqrt{\frac{\varepsilon_{f s}}{\varepsilon_{m p}}}
$$

In direct sending, each sensor sends the data directly to the center. Direct sending networks are very simple and easy to design, however due to long distance of the sensors from the central station; they consume a lot of energy. On the contrary, designs that shorten the communication distance can prolong life cycle of the network. Due to high congestion of sensor nodes in surface unit and because of their proximity to each other, multi-step communication in such networks are more useful and more commodious than single-step communications. Where $\varepsilon$ is signal strengthening factor and Eelec is energy consumption during transfer and receiving. For receiving 1 bit data message in the distance of $d$, the amount of radio consumption is based on (3) [10]:

$$
E_{R X}(l)=l \times E_{\text {elec }}
$$

\subsection{Description of the proposal method}

The proposed method in this project has two main phases called initiation phase and data transfer phase. Initiation phase includes head cluster selection, cluster formation and creation of minimum spanning tree between head clusters and in data transfer phase, the data are sent through the created routes.

\subsubsection{Initiation phase}

This phase is composed of the threesteps.

\section{Step 1: Selection of head cluster}

This step is similar to LEACH algorithm. During this step, clusters are formed using a distributed algorithm. Each sensor node generates a random number between $0 \sim 1$, without any control from central station. If the randomly generated number is lower than the threshold limit $T(n)$, that node is considered as head cluster. $T(n)$ is obtained using equation (4)

$$
T(n)=\left\{\begin{array}{cc}
\frac{p}{1-p \times\left[\operatorname{rmod}\left(\frac{l}{p}\right)\right]}, & n \in G \\
0, & \text { otherwise }
\end{array}\right.
$$

whereP is probability of becoming a head cluster, $r$ is the current round, and $r \bmod \left(\frac{l}{p}\right)$ is the number of nodes selected as head cluster during $r$ round. Letter $G$ stands for a set of nodes that are not selected as head cluster during round $r$, and $n$ is the total number of nodes.As nodes had an equal energy at the beginning, nodes that have not been head cluster recently, definitely have more energy left, compared to other nodes and it is better to be selected as head cluster during next rounds. After all nodes are once selected as head cluster, all nodes meet the criteria to become a head cluster during next rounds.

\section{Step 2: Cluster formations}

After selecting head cluster, head cluster node must inform other network nodes of its roles as a head cluster, so that the nodes can select it as head cluster during the current round. So, each head cluster node, broadcast an ADV message, using CSMA (Mac) protocol within the whole network. ADV message contains id of head cluster node and location data of the head cluster. After that, non-head cluster nodes select a head cluster based on1) less distance to them, 2) much more 
energy. At this time it is necessary that each node informs its selected head cluster that it is about to join that cluster. So each sensor node sends a Join-Request message (including id of node and id of head cluster node) using CSMA (Mac) protocol to the related head cluster.Head cluster node allows other cluster nodes to transfer data in particular time divisions, using TDMA (Time Division Multiple Address) schedule. This schedule guarantees that no conflict happens among the data messages, also allows the radio components of non-head cluster nodes to sleep at all times except during data transfer times, which reduces energy consumption in sensors [11]

\section{Step 3: Creation of minimum spanning tree between the head clusters}

After creating the cluster, head cluster nodes send an ADV message to central station which includes head cluster id, location details of head cluster, number of nodes connected to cluster and remained energy of the head cluster. Central station creates a minimum spanning tree based on the information received from head clusters including the geographical location of the cluster heads and their remained energy.To create the spanning three we apply the well-known algorithm Prime [15] by considering the multiplication of remained energy of the candid cluster head and its distance as the weight of the edges. Then the tree structurebroadcasts to head clusters and they communicate with central station through the shortest routes connected to each other.In creation of the proposed minimum spanning tree, data aggregation is also performed. That is, each parent node, after receiving data frames from head clusters connected to it, combines them together and removes repetitive data, then sends the aggregated data to its upper parent. This process continues until the aggregated data reach the root of tree, i.e., the central station [12].

\subsubsection{Data transfer phase}

During this phase, member nodes send a cluster to head cluster, along their specific time division and without any interference in data frame network. The time period, in which every node transfers data is fixed, therefore time of sending a frame depends on number of the nodes in cluster. To reduce loss of energy, each non-head cluster node sends data by controlling the power, therefore radio component of each non-head cluster node is off until time division of data transfer is assigned to it. However radio component of head cluster node must be always active to aggregate data from nodes that are member of its local cluster. When the head cluster receives all data related to nodes of its cluster, performs data aggregation on them and creates a proper data package. Then, through the tree structure which is formed between head clusters, sends its package of aggregated data to parent. Each parent, after performing data aggregation on its own local data as well as the data received from its child sensors, sends them to upper level. In the proposed method, central station is considered as root of the tree which ultimately received aggregated data from upper parents.

\section{SIMULATION ENVIRONMENT}

Simulation environment is similar to LEACH protocol [14] and the objective is to save energy and optimize energy consumption. In this experiment, 100 sensor nodes are scattered randomly in a certain region of $100 \times 100$. Central station is far from the sensors, located at coordinate $(50,200)$. Figure 1 shows this setting up.
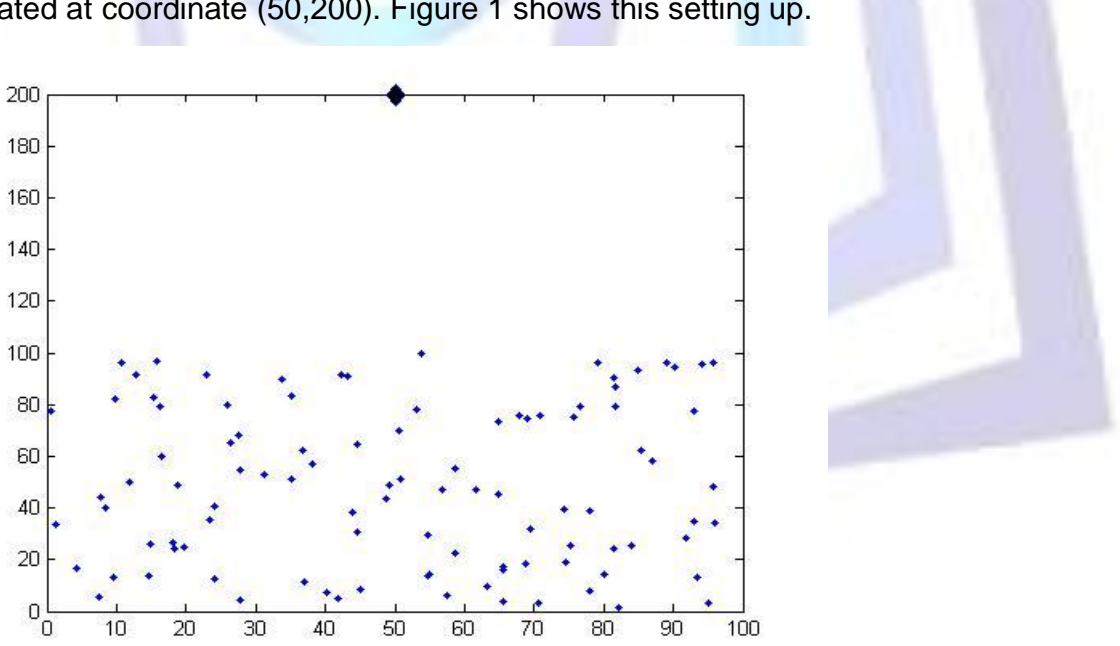

\section{Fig 1: Hundred sensor nodes randomly scattered}

Primary energy of the 100 sensor nodes is equal in this simulation, and each sensor node has an initial energy of $0.5 \mathrm{~J}$ at the beginning. Central station is a high power node, capable of receiving unlimited amount of data. $E_{\text {elec }}$ is energy consumption during transfer and reception, which is considered to be 50nj/bit here. Energy consumption in Free Space channel model (fs) is equal to $10 \mathrm{PJ} / \mathrm{bit} / \mathrm{m} 2$ and energy consumption is Multipath induced fading model (mp) is equal to $0.0013 \mathrm{PJ} / \mathrm{bit} / \mathrm{m} 4$. The amount of energy required for data aggregation in cluster head nodes (EDA) is considered to be $5 \mathrm{~nJ} / \mathrm{bit} / \mathrm{signal}$. Size of Control data packet (C) which is exchanged to develop cluster, announce cluster head, TDMA timing and developing Minimum Spanning Tree between network nodes, is equal to 800 bits. Length of data bundles that includes data sensed from environment and sent by cluster nodes and head cluster node, is equal to 4000 bits. Simulation time is 100 rounds and during each round 5 frames of data is sent. In short, parameters used in simulation of the network are given in Table 1. 
Table 1. Parameters used in the simulation of network

\begin{tabular}{|c|c|}
\hline Value & Parameter \\
\hline $0.5 \mathrm{~J}$ & Initial energy \\
\hline $50 \mathrm{~nJ} / \mathrm{bit}$ & Eelec= ETX = ERX \\
\hline $10 \mathrm{PJ} / \mathrm{bit}^{\mathrm{m}} \mathrm{m}^{2}$ & $\begin{array}{c}\text { Enery consumption in free space } \\
\text { model (fs) }\end{array}$ \\
\hline $0.0013 \mathrm{PJ} / \mathrm{bit}^{\mathrm{m}} \mathrm{m}^{4}$ & $\begin{array}{c}\text { Energy consumption in Multipath } \\
\text { induced fading model(mp) }\end{array}$ \\
\hline $5 \mathrm{~nJ} / \mathrm{bit} / \mathrm{signal}$ & Energy dataaggregation (EDA) \\
\hline $800 \mathrm{bit}$ & Control packet size \\
\hline $4000 \mathrm{bit}$ & The size of the data packet \\
\hline $130 \mathrm{round}$ & Time simulation \\
\hline
\end{tabular}

Total number of nodes that survive during simulation is compared consideringthe standard LEACH protocol, $f$ weightedLEACH and Weighted-TLEACH methods. Figure 2 shows the live nodes in simulation, and indicates that the number of the live nodes during simulation in Weighted-TLEACH is more than that of Weighted-LEACH and LEACH protocol. Also, It can be seen the weighted methods generally outperform the standard LEACH method.

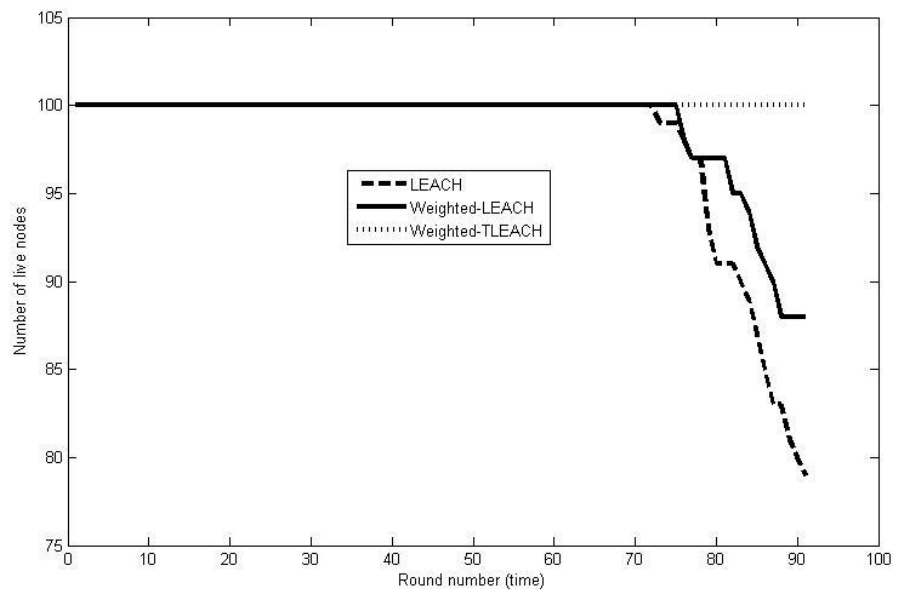

Fig 2: Number of sensors surviving during network simulation

Figure 3 shows total amount of energy consumption in Weighted-TLEACH, Weighted-LEACH and LEACH protocol Comparison of energy consumption of methods shows that the objective of reducing energy consumption in WeightedTLEACH is fulfilled.

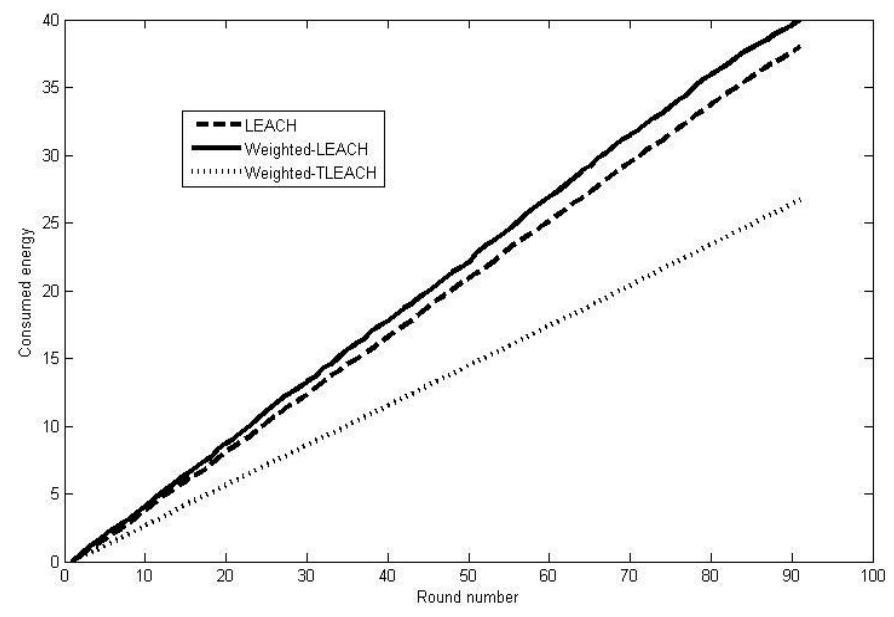

Fig 3: Total energy consumption in the two proposed methods and LEACH protocol during simulation 
Figure 4 shows variance of energy of each node in three methods. Naturally, the higher the energy variance in a method, the more incongruent is that method. Energy variance of each node in the proposed method of Weighted-TLEACH is much less than Weighted-LEACH and LEACH.

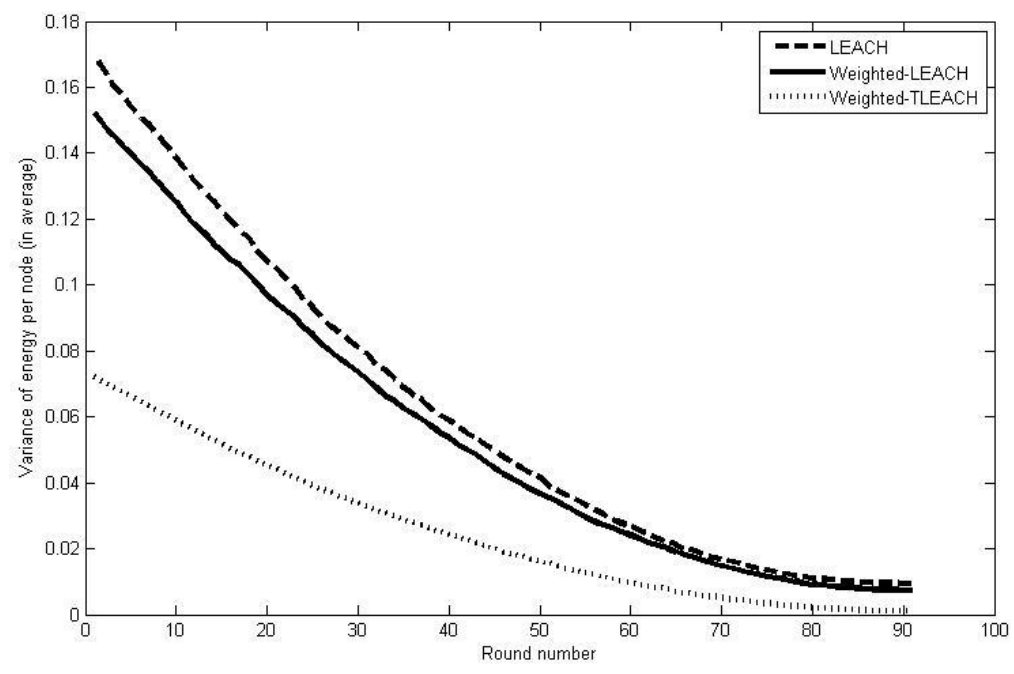

Fig 4: Variance of energy of each sensor node in three simulation methods

The top of Figure 5 shows the number of head clusters in every round, in three methods. Regarding the fact that clustering algorithm is the same for all three methods, thus distribution of the clusters is similar in all three methods.

The bottom of Figure 5 shows the amount of energy consumption in each round. Oscillation of cluster changes in each round is given in this graph, too. However, oscillation of energy consumption in Weighted-TLEACH is less than the other two methods which shows that number of clusters has no much impact on energy consumption.
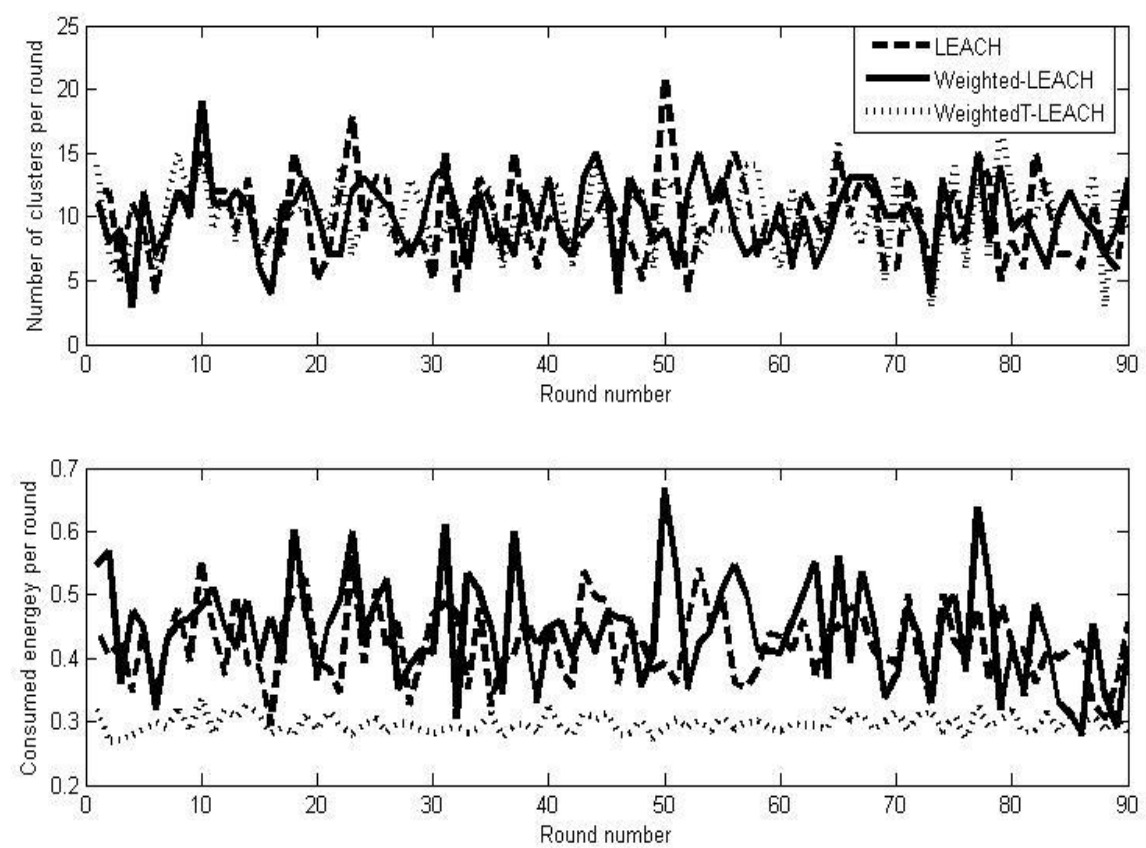

Fig 5: Number of 5 head clusters and energy consumption in each round

Figure 6 shows graph of energy distribution in the three methods, during simulation. Dark color in this graph represents the highest level of energy and bright colors are lowest level of energy and the energy at zero level. The left part of Figure 6 which is related to LEACH protocol shows that this fact that since all sensors in LEACH have the chance of becoming a head cluster, energy of all sensors during the time $s$ almost the same and there is no significant energy change among different sensors. The middle part and right part of Figure 6 demonstrate the the graph of energy distribution ofWeightedLEACH and Weighted-TLEACHmethods respectively. As can be seen the energy distribution of the LEACH and Weighted-LEACH are th almost the same energy distribution, but energy distribution of Weighted-TLEACH method is more uniform $r$ than others. 

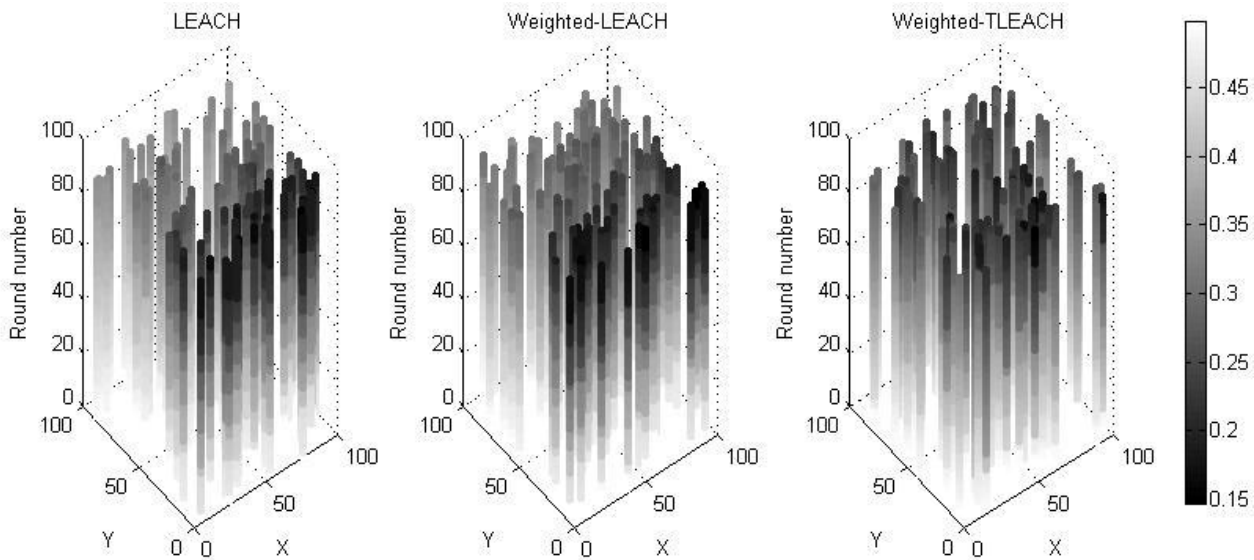

Fig 6: Graph of energy distribution

\section{CONCLUSION AND FUTURE WORKS}

In this paper we study the holes problem in wireless sensor networks. For this purpose, a heuristic method is proposed that takes attention to the available energy of each node as an important parameter during the formation of clusters and the minimum spanning tree. The performance of the proposed method discussed through extensive simulations. As a future work, we will consider other important parameters such as the number of clusters, duration of each round of the data aggregation algorithm that can effect the holes problem.

\section{ACKNOWLEDGMENTS}

We are appreciate to thanks Islamic Azad university-Omidieh branch for their fund support of this research.

\section{REFERENCES}

[1] Akyildiz, I. F. Su, W. 2002. Wireless Sensor Networks: A Survey. J. Computer Networks, vol. 38, pp. $393-422$.

[2] Kahn J.M., Katz R.H. and Pister K.S.J. 1999. Next century challenges: mobile networking for smart dust.in: Proceedings of the ACM MobiCom 99, Washington, USA, pp. 271-278.

[3] Ilyas M. and Mahgoub I.2005. Handbook of Sensor Networks: Compact Wireless and Wired Sensing Systems.In: Proceedings of the CRC Press, London, Washington, D.C.,

[4] Rajagopalan, R. Varshney, P. K. 2006. Data Aggregation Techniques in Sensor Networks: A Survey, J. IEEE Communications Surveys \& Tutorials, vol. 4, pp. 48-63.

[5] Nandini. S. P, Prof. P. R. P. 2011.Data Aggregation in Wireless Sensor Network. IEEE International Conference on Computational Intelligence and Computing Research.

[6] Jamal N. Al-Karaki. 2009.Data aggregation and routing in Wireless Sensor Networks: Optimal and heuristic algorithms. Computer Networks 53.

[7] Abbasi. A.A, Younis. M. 2007.A survey on clustering algorithms for wireless sensor networks, Computer Communications.

[8] Krishnamachari, B. Estrin, D. and Wicker, S. 2002. The Impact of Data Aggregation in Wireless Sensor Networks. Proc. 22nd Int'l. Conf. Distributed Computing Systems Wksps, pp. 575-78,July.

[9] Heinzelman, W. B. Chandrakasan, A. P. Balakrishnan, H. 2002. An Application Specific Protocol Architecture For Wireless Microsensor Networks. J. IEEE Transactions on Wireless Communications,vol. 4,pp. 660-669.

[10] Heinzelman, W. Chandrakasan, A. Balakrishnan, H. 2000. Energy-Efficient Communication Protocol for Wireless Microsensor Networks. In Proc. of IEEE International Conference on System Sciences 2000, pp. 1-10.

[11] Wang, W. Wang, B. 2011. Cluster-based And Tree-based Power Efficient Data Collection and Aggregation Protocol for Wireless Sensor Network. J. Information Technology, vol. 10, pp. 557-564.

[12] Marc, L. Vincent, W. 2005. An Energy-Aware Spanning Tree Algorithm for Data Aggregation in Wireless Sensor Network, IEEE.

[13] Manish, K. Shekhar, V. Pankaj, P. 2008. Clustering Approach to Data Aggregation in Wireless Sensor Networks, IEEE ICON.

[14] Lutful, K. Nidal, N. 2010. An Efficient Data Aggregation Approach for Large Scale Wireless Sensor Networks, Communications Society subject matter experts for publication in the IEEE Globecom 2010.

[15] Abhilasha, R. 2013. Minimum cost spanning tree using Prim's algorithm.International journal of advance Research in computer science and management studies, 1(1). 